\title{
Consolidación de los productos de madera de la organización social Alianza Selva Maya, Quintana Roo con la aplicación de herramientas de márquetin
}

\section{Consolidation of the wood products of the social organization Alianza Selva Maya, Quintana Roo with the application of marketing tools}

DOI: $10.46932 / \mathrm{sjjdv} 3 \mathrm{n} 1-055$

Received in: Jan 30st, 2021

Accepted in: Feb 1th, 2022

\section{Dr. Jorge Antonio Torres Pérez}

Profesor - Investigador de la División de Ciencias Forestales de la Universidad Autónoma Chapingo.

E-mail: jorgeatorresperez@yahoo.com.mx

\section{Dra. Zazil Ha Mucui Kac García Trujillo}

Profesora del Instituto Tecnológico de la Zona Maya. Juan Sarabia, Othón P. Blanco, Chetumal, Quintana Roo.

E-mail: zazilgarcia@gmail.com

\section{M.C. Martha Alicia Cázares Morán}

Profesora del Instituto Tecnológico de la Zona Maya. Juan Sarabia, Othón P. Blanco, Chetumal, Quintana Roo.

E-mail: acm0629@gmail.com

\section{M.C. Alicia Avitia Deras}

Profesora del Instituto Tecnológico de la Zona Maya. Juan Sarabia, Othón P. Blanco, Chetumal, Quintana Roo.

E-mail: avitiaderas@ hotmail.com

\section{Lic. Cecilia Loría Tzab}

Profesora del Instituto Tecnológico de la Zona Maya. Juan Sarabia, Othón P. Blanco, Chetumal, Quintana Roo.

E-mail: loria.t.cecilia@gmail.com

\section{Ing. Claudia Palafox Bárcenas}

Directora ejecutiva de Tropica Rural Latinoamericana A. C.

E-mail: claudia.palafoxbarcenas@gmail.com

Ing. Yuriria Hernández Velasco

Estudiante de Ingeniería Forestal del Instituto Tecnológico de la Zona Maya

E-mail: i18870086@zonamaya.tecnm.mx

\section{RESUMEN}

La Alianza Selva Maya Quintana Roo UE de RL (UEASM), integra 5 ejidos forestales de Quintana Roo, estos son responsables del manejo forestal desde 1983. Tienen 113,000 hectáreas bajo manejo forestal sustentable y 66,321 hectáreas certificadas bajo estándares internacionales de FSC. La Iniciativa Industrial de la UEASM, denominado K’áax Mayas, desarrolla la producción de muebles y pisos de madera sólida enfocada al mercado de la Riviera Maya. Con el presente proyecto, se apoya a la UEASM en el tema de 
mercadeo y comercialización, ya que no basta con tener una excelente producción, sino se concretan en ventas. El objetivo de UEASM es impulsar el comercio y beneficiar los ejidatarios que aprovechan los recursos forestales alineados con las prácticas realizadas tradicionales. Este proyecto aborda temas de mercadotecnia, para mejorar los procesos de comercialización bajo esquemas de comercio justo y economía solidaria y diseña alternativas innovadoras de comercialización, basada en plataformas digitales. Actualmente seis de cada 10 mexicanos se enteran de un producto nuevo por publicidad en Internet y siguen las redes sociales para decidir su compra. Por lo anterior es importante que la UEASM incursione en los sistemas de mercadotecnia digital para posicionar su marca y productos en los mercados.

Palabras clave: empresa rural, productor forestal, desarrollo comunitario, mercado justo, publicidad digital.

\begin{abstract}
The Alianza Selva Maya Quintana Roo UE de RL (UEASM), integrates 5 forest ejidos in Quintana Roo, which have been responsible for forest management since 1983. They have 113,000 hectares under sustainable forest management and 66,321 hectares certified under FSC international standards. The UEASM Industrial Initiative, called K'áax Mayas, develops the production of solid wood furniture and flooring focused on the Riviera Maya market. This project supports UEASM in marketing and commercialization, since it is not enough to have an excellent production, but also to have sales. UEASM's objective is to promote trade and benefit the ejidatarios who use forest resources in line with traditional practices. This project addresses marketing issues to improve marketing processes under fair trade and solidarity economy schemes and designs innovative marketing alternatives based on digital platforms. Currently, six out of 10 Mexicans learn about a new product through Internet advertising and follow social networks to decide their purchase. Therefore, it is important for UEASM to venture into digital marketing systems to position its brand and products in the markets.
\end{abstract}

Keywords: rural enterprise, forest producer, community development, fair market, digital advertising.

\title{
1 INTRODUCCIÓN
}

La riqueza forestal de México, que en su mayoría pertenece a ejidos y comunidades, genera productos y servicios ambientales que contribuyen a la economía nacional. No obstante, de esta riqueza, la actividad forestal no se ha reflejado en el desarrollo sustentable de la población rural. Contrariamente se han provocado diversos problemas: degradación de recursos forestales, cambio de uso de suelo, tala clandestina, incendios forestales y deforestación.

Uno de los estados que han desarrollado la actividad forestal es Quintana Roo, el cual, a inicios de los años 80 implementó el Plan Piloto Forestal (PPF), como estrategia gubernamental para regular los aprovechamientos comunitarios. Una de las estratégicas fue que las comunidades establecieran Áreas Forestales Permanentes (AFP), que consistió en declarar un área exclusivamente para aprovechamiento forestal por lo que no podía tener cambio de uso de suelo. Esta estrategia ayudó a la conservación de grandes macizos forestales que hasta la fecha se conservan. 
El manejo forestal ejidal se fue desarrollando en diversos niveles en la región. El PPF sentó las bases de regulación y los ejidos tomaron los acuerdos para los aprovechamientos. No cabe duda que hubo una apropiación del recurso natural y que se crearon las condiciones para que las comunidades aprendieran sobre la marcha.

Algunos ejidos han hecho esfuerzos por diversificar la actividad económica y avanzaron en darle valor agregado a la madera poniendo aserraderos, estufas de secado y carpinterías en los ejidos, sin embargo se enfrentaron a la problemática de poder abastecer contratos con volúmenes grandes, poder tener el volumen apropiado de madera de una sola especie para el secado en las estufas industriales, las limitaciones de producción, la poca experiencia especializada en carpintería y la falta de equipos para desarrollar productos de madera, impiden que estas comunidades creen productos de alta calidad que les permita insertarse en el creciente mercado regional de la Riviera Maya del Caribe Mexicano.

Como estrategia conjunta se establece la Unión de Ejidos “Alianza Selva Maya de Quintana Roo UE de RL" (UEASM) integrada por cinco ejidos forestales -Bacalar, Petcacab, X-Hazil, Noh-Bec y Felipe Carrillo Puerto, en donde habitan 1,237 ejidatarios con sus familias, la mayoría de origen maya y con derechos agrarios. Dos de los ejidos tienen certificado FSC® de manejo forestal responsable -Ejido Petcacab (FSC-C136354) y Ejido Noh-Bec (FSC-C018252)-, que juntos suman 66,321 hectáreas certificadas.

Es por lo anterior, que la UEASM, emprendió la tarea de aprender cómo desarrollar la industria de la madera, asumiéndose como una empresa social forestal, con la idea de construir un ejemplo regional que incentivara a los ejidos forestales a invertir en esta industria, pero con alto valor agregado, basada en la belleza de las maderas nativas de la selva maya. El emprendimiento industrial de la UEASM, conocido como Iniciativa Industrial K’áax Mayas, está desarrollando la producción de muebles y pisos de madera sólida enfocada en el mercado de la Riviera Maya.

Esta organización desarrolló la capacidad local de producir muebles en las comunidades mayas. Por un lado, en la UEASM se formó un grupo de carpinteros de alto nivel que se encarga del desarrollo de productos enfocados en el mercado regional, y por el otro, con el apoyo de diversas instituciones nacionales e internacionales, se instalaron dos carpinterías artesanales, una ubicada en el Instituto Tecnológico de la Zona Maya y la otra en el ejido de Nohbec, entrenando carpinteros y habilitando secadoras solares para madera.

La iniciativa está trabajando, sin embargo no se han abordado completamente los aspectos de mercadeo, que se puede identificar como el último eslabón de la cadena productiva, por lo que la Universidad autónoma Chapingo (UACh) en vinculación con la UEASM está trabajando un proyecto que tiene como objetivo diseñar estrategias de mercadeo, comercialización y de mercadotecnia para los 
productos ofertados. Con la implementación del presente proyecto, se pretende apoyar a la UEASM en el tema de mercadeo, comercialización y mercadotecnia de sus productos, ya que no basta con obtener una excelente producción sino se concretan en ventas, con las cuales los ingresos se reflejen en la rentabilidad de esta iniciativa. Se presentan los primeros avances de esta investigación, ya que esta continuará para desarrollar todos los elementos planteados en los objetivos específicos de la investigación.

\section{ANTECEDENTES}

La Alianza Selva Maya Quintana Roo UE de RL (UEASM), integra a 5 ejidos forestales de Quintana Roo, estos ejidos son sujetos responsables del manejo forestal desde 1983, todos han pasado un ciclo de corta de 25 años y permanecen en la forestería comunitaria como forma de vida. En su conjunto estos cinco ejidos tienen 215,434 ha, de las cuales 113,000 están bajo manejo forestal sustentable y 66,321 hectáreas certificadas bajo estándares internacionales FSC (Acta constitutiva de la UEASM, 2014). Los ejidos organizados reconocen la necesidad de agruparse para enfrentar los nuevos retos que implica la certificación del manejo forestal, el acceso a los mercados y la defensa de la forestaría comunitaria como modo de vida rural, esto incluye la participación de la Universidad Autónoma Chapingo (UACh) como promotor de proyectos en apoyo a los ejidos participantes en la alianza.

La integración del sector académico a esta alianza fortalece la participación y aprendizaje de los alumnos para tener una vinculación con los sectores productivos de las selvas tropicales del Estado de Quintana Roo. La alianza Selva Maya además de fortalecer la forestería comunitaria, ha incorporado proyectos de ecoturismo y de captura de carbono, y ha desarrollado técnicas de adaptación agrícola para el cambio climático, lo que representa oportunidades de un desarrollo diversificado.

El objetivo de la vinculación académica con el sector productivo va encaminada al impulso de la organización social, empresarial y conservación de los recursos naturales como parte de la formación integral de nuestros estudiantes a través de la interacción con organizaciones nacionales e internacionales que son actores activos en estos rubros.

La Alianza Selva Maya Quintana Roo UE de RL (UEASM) tiene como objetivo impulsar el comercio y beneficiar a los ejidatarios que aprovechan los recursos forestales tropicales del sureste de México en línea con lo que han realizado productivamente por tradición.

La UEASM ofrece productos maderables de maderas tropicales certificadas con los principios del FSC. Promueve el desarrollo de la forestaría rural comunitaria basado en el manejo responsable de los recursos forestales a través de la iniciativa industrial K’áax Mayas y que esta se convierta en un canal de comercialización para lo cual este proyecto brinda asesoría es temas de mercadeo, canales de comercialización alternativos y aspectos de mercadotecnia, a través de la mejora de los procesos de 
comercialización bajo esquemas de comercio justo y economía solidaria impulsando los procesos de transformación de productos forestales y diseñando alternativas innovadoras de procesos de comercialización basada en plataformas digitales.

\section{METODOLOGÍA}

\subsection{UBICACIÓN DE LA ZONA DE ESTUDIO}

Los ejidos que integran la UEASM se encuentran en los municipios de Felipe Carrillo Puerto y Bacalar en el estado de Quintana Roo, dentro del bloque forestal conocido como el corredor biológico mesoamericano. En la figura 1 se presenta la ubicación de los cinco ejidos.

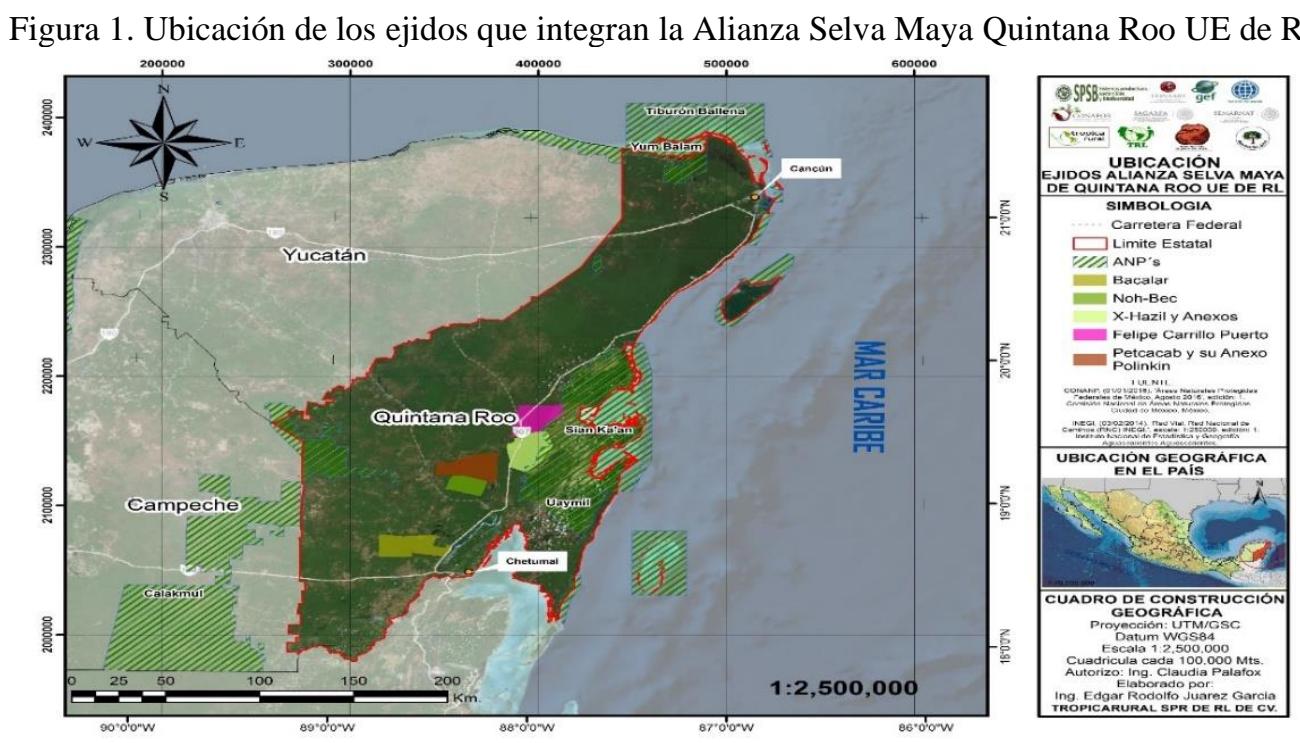

La primera etapa del estudio consistió en el análisis de los productos ya desarrollados y una investigación de las posibilidades para promoción y ventas como estrategia de entrada en el mercado.

Como primer acción se realizó un taller participativo con los directivos de la organización UEASM para identificar y definir los alcances del proyecto para que los productores y los profesores participantes tuvieran claro los resultados esperados.

Se realizó una investigación para diseñar la estrategia comercial en donde se analizaron las opciones de promoción de los productos: tiendas propias; tiendas de cadenas en plazas comerciales, televisión, radio, revistas, periódico, publicidad externa y ventas por internet, mediante el método de matriz de comparación y priorización se seleccionaron los factores a evaluar para poder tener putos de referencia para la selección de las estrategias de promoción. En el cuadro 1 se presentan los factores que a evaluar. 
Cuadro 1. Definición de factores a evaluar

\begin{tabular}{|l|l|}
\hline FACTOR & DESCRIPCIÓN \\
\hline Costo & Precio que se debe pagar por el medio utilizado \\
\hline Accesibilidad & Facilidad o dificultad de acceso al público meta del medio publicitario utilizado \\
\hline Facilidad de uso & Si requiere o no asesoría técnica, capacidad de interpretar y tiempo u horario de consulta \\
\hline Credibilidad & $\begin{array}{l}\text { Confiabilidad del publico meta en la información que recibe (calidad y prestigio del } \\
\text { medio utilizado) }\end{array}$ \\
\hline Participación & $\begin{array}{l}\text { Facilidad de participación del público meta para realizar comentarios y preguntas y } \\
\text { resolver dudas }\end{array}$ \\
\hline Difusión del mensaje & Magnitud de la impresión, tiempo del mensaje y vida del mensaje en el medio utilizado \\
\hline $\begin{array}{l}\text { Relación con los } \\
\text { objetivos }\end{array}$ & Forma en que el medio cumple más adecuadamente con el objetivo publicitario \\
\hline Alcance & No. de personas a las que se llega por el medio publicitario \\
\hline Rastreo del cliente & Facilidad para identificar, ubicar, comunicarte con tu cliente y darle seguimiento \\
\hline
\end{tabular}

Con los resultados de los factores de promoción se identificaron y seleccionaron los servicios que se ofrecen en línea, que brinde la infraestructura para la venta y promoción de productos en línea a través de una matriz de comparación y priorización. A cada factor se le evaluó mediante una ponderación en una escala de valores.

\section{RESULTADOS}

El taller participativo con los directivos de la organización dio como resultado los alcances generales de la organización y los alcances del proyecto, los cuales incluyen aspectos se sociales y comerciales entre los que destacan: Mejorar el modo y calidad de vida de las familias que integran los ejidos forestales, como estrategia de desarrollo forestal comunitario. Mantener la cobertura forestal de 160 mil ha. Mantener altos valores de conservación comunitaria. Diversificar actividades productivas. Diversificar productos, cubriendo la demanda de los diferentes mercados existentes y emergentes. Contar con un equipo humano capacitado, sensibilizado y comprometido con la cultura de servicio al cliente

En los aspectos comerciales destacan: Comercializar el 100\% del volumen total autorizado buscando mercados de productos certificados y satisfaciendo una demanda constante de madera estufada. Arraigar la Cultura de Servicio al Cliente como base del desempeño de todos y cada uno de los miembros de los ejidos y de las Empresas Forestales. Contar con un portafolio de productos derivados de las maderas tropicales que maximice el valor ofrecido al cliente, minimizando sus costos de transacción y maximizando su satisfacción. Diseñar una estrategia digital de Social Media. Diseño de una tienda on line. Capacitar a los socios de la UEASM en el Manejo Social Media. Capacitar a los responsables de la comercialización en el manejo y actualización de la estrategia de mercadeo, comercialización y mercadotecnia. 


\subsection{ESTRATEGIA COMERCIAL}

En los últimos años se han presentado cambios radicales en la forma de hacer negocios, las tecnologías nuevas han obligado a transformar las expectativas de una marca, así como la manera de consumir nuevos y diversos servicios. Actualmente no es suficiente tener puntos de venta fijos, pues seis de cada 10 mexicanos se enteran de un producto nuevo por publicidad en Internet y siguen los comentarios en redes sociales para decidir su compra, según un estudio hecho por IAB México y la firma Millward Brown (2018). La tecnología cambia los hábitos de los consumidores, quienes buscan hacer compras no sólo en los horarios de las tiendas, sino incluso sin salir de su casa u oficina. Esta es una tendencia que también modifica las estrategias de venta de los comercios (. Por lo anterior es importante que la UEASM incursione en los sistemas de mercadotecnia digital para posicionar el concepto y productos en los marcados. En México, el comercio electrónico crece 15 veces más que la economía y, al menos por ahora, no se ve un techo que pare esa tendencia, aseguran expertos en el tema consultados por La Jornada (Gutiérrez Julio, 2019).

La primera línea de producción fue enfocada a la producción de duela y deck de madera y muebles de diseño y sobre diseño requerido por el cliente, juegos didácticos y artículos decorativos para el hogar. Se presentan imágenes de la primera línea de productos. En las figuras 2, 3, 4 y 5 se muestran imágenes de los productos.

Figura 2. Sillas y organizadores.

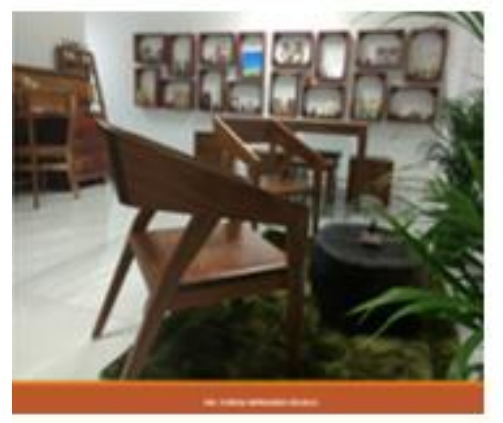

Figura 3. Organizadores y sillas.

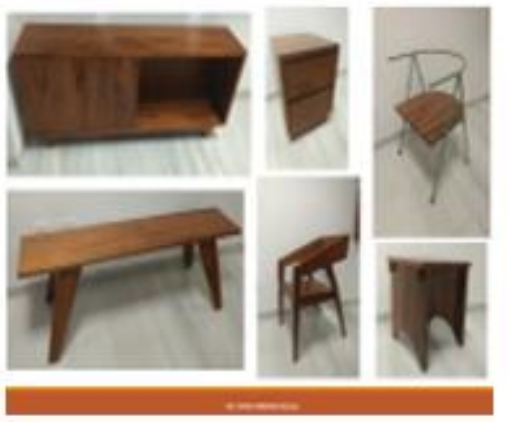

Figura 5. Juegos y decoración

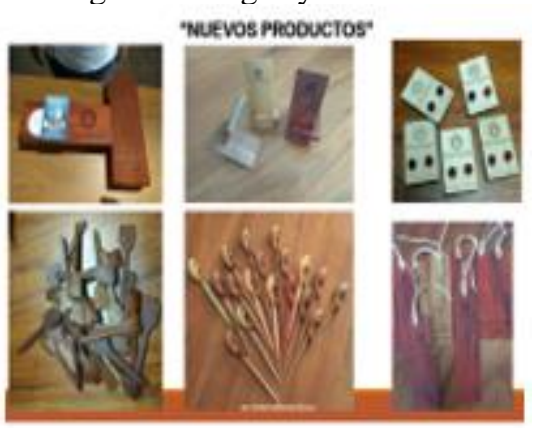

Para la estrategia comercial se diseñó una marca que identificara el concepto de selva maya con los productos, se registró como K’áax Mayas y se realizaron los trámites de registro. En la figura 6 se presenta el logotipo y en la figura 7 el registro de marca. 
Figura 6. Diseño y marca registrada

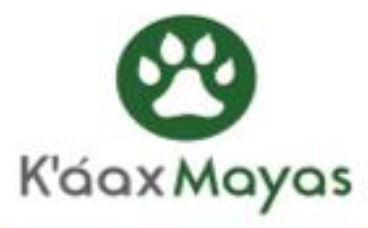

Figura 7. Registro de marca
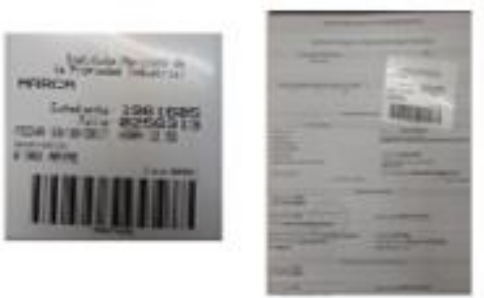

De acuerdo a Kotler (2008), entre las cualidades deseables de un nombre de marca están: Debe sugerir algo acerca de los beneficios y cualidades del producto. Debe ser fácil de pronunciar, reconocer, y recordar. Los nombres cortos son buenos. El nombre de marca debe ser distintivo. Debe ser fácil traducir el nombre a otros idiomas. Debe poder registrarse para protegerlo legalmente.

\subsection{ESTRATEGIA INICIAL DE COMERCIALIZACIÓN DE LA UEASM}

Se han definido inicialmente los puntos de venta estratégicos que permitan al mismo tiempo la venta y mostrar los productos. Los puntos de venta se ubican en el aeropuerto de Chetumal Quintana Roo, Hotel Fiesta Inn y el Callejón del arte.

Se abrieron dos puntos de venta: el ShowRoom K'áaxMayas en el Hotel Villanueva en Chetumal, Quinta Roo y un stock en la Galería del Restaurante el Manatí en Bacalar, Quinta Roo. Kotler (2012) menciona que A pesar de que la mayoría de las ventas al menudeo se realizan en tiendas, en años recientes estas ventas fuera de las tiendas han crecido con mayor rapidez que las ventas dentro de las tiendas. Las ventas al menudeo fuera de las tiendas incluyen las ventas a consumidores finales por Internet, correo directo, catálogos, teléfono u otros métodos de ventas directas.

La estrategia en redes sociales está basada en plataformas establecidas y que fueron elegidas de acuerdo al impacto social que tienen. La más fuerte hasta el momento es el Facebook en donde se interactúa directamente con los clientes potenciales y se presentan los productos nuevos. En la figura 8 se presenta la imagen corporativa en la red social.

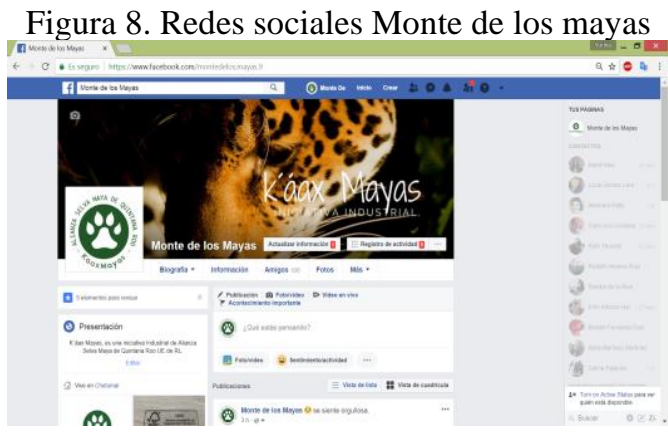


Matriz de evaluación para decidir medio para promover los productos de Alianza Selva Maya Quintana Roo UE de RL K'áaxMayas.

La UEAS ya inicio con distribución física de los productos que oferta a través de sitios de venta. Se está analizando la posibilidad de buscar colaborar con algunas tiendas de prestigio para como Liverpool, Palacio de Hierro, así como otras estrategias de venta como establecer puntos de venta de la entrada a todas las terminales marítimas del estado de Quintana Roo (muelle de Mahahual, Playa del Carmen, Cozumel, Isla Mujeres y Cancún) o establecer una tienda propia en las principales plazas comerciales de la Riviera maya, cuidando los costos que esto implica y abrir una tienda on line y las mejorar las estrategias de venta por internet explorando las diferentes opciones.

Para la toma de decisiones de los medios de promoción se analizaron seis opciones de promoción y se analizaron con los factores de ponderación presentados. Esta nos señala la importancia de cada factor de acuerdo a los intereses empresariales. Se presentan los resultados de la matriz de evaluación en donde se calificaron los medios de promoción con los factores y se aplicó la ponderación. En el cuadro 2 se muestra que con base a los resultados, el mejor medio de promoción fue el internet (página web).

Cuadro 2. Matriz Producto Calificación * Ponderador

\begin{tabular}{|c|c|c|c|c|c|c|c|c|c|c|}
\hline $\begin{array}{l}\text { MEDIO/FACT } \\
\text { OR }\end{array}$ & $\begin{array}{l}\text { COS } \\
\text { TO } \\
\end{array}$ & $\begin{array}{l}\text { ACC } \\
\text { ESIBI } \\
\text { LIDA } \\
\mathrm{D} \\
\end{array}$ & $\begin{array}{l}\text { FACILI } \\
\text { DAD } \\
\text { DE } \\
\text { USO } \\
\end{array}$ & $\begin{array}{l}\text { CRE } \\
\text { DIBI } \\
\text { LIDA } \\
\mathrm{D} \\
\end{array}$ & $\begin{array}{l}\text { PARTI } \\
\text { CIPA } \\
\text { CIÓN }\end{array}$ & $\begin{array}{l}\text { DIFUSIÓ } \\
\text { N DEL } \\
\text { MENSAJ } \\
\text { E }\end{array}$ & $\begin{array}{l}\text { RELACIÓ } \\
\text { N CON } \\
\text { LOS } \\
\text { OBJETIVO } \\
\text { S } \\
\end{array}$ & $\begin{array}{l}\text { ALCA } \\
\text { NCE } \\
\end{array}$ & $\begin{array}{l}\text { RASTRE } \\
\text { O DEL } \\
\text { CLIENTE }\end{array}$ & $\begin{array}{l}\text { SUM } \\
\text { ATO } \\
\text { RIA } \\
\end{array}$ \\
\hline Televisión & 4 & 8 & 3 & 12 & 25 & 12 & 6 & 4 & 25 & 74 \\
\hline $\begin{array}{l}\text { Internet } \\
\text { (página web) }\end{array}$ & 20 & 4 & 3 & 12 & 5 & 6 & 4 & 4 & 5 & 58 \\
\hline Revistas & 12 & 16 & 6 & 8 & 25 & 12 & 6 & 16 & 20 & 101 \\
\hline Periódico & 16 & 8 & 6 & 8 & 25 & 9 & 8 & 16 & 25 & 96 \\
\hline $\begin{array}{l}\text { Publicación } \\
\text { exterior }\end{array}$ & 8 & 12 & 9 & 12 & 25 & 12 & 6 & 16 & 25 & 100 \\
\hline Radio & 16 & 8 & 3 & 12 & 20 & 12 & 6 & 8 & 25 & 85 \\
\hline
\end{tabular}

Con relación a la estrategia de mercadotecnia por internet, e-commerce y el Dropshiping son dos alternativas para abrir posibilidades de ventas de los productos de UEASM. David Díaz, responsable de consumo masivo de la firma digital MercadoLibre, indica después de 20 años de funcionamiento, la cantidad de clientes aumenta, en línea con una mayor demanda de productos y servicios, incluidos los financieros. Menciona que en México han alcanzado 25 millones, lo cual corresponde a un tercio de la gente con Internet en el país. Comenta que parte de la evolución de ese mercado obedece a la transformación que se ha tenido en temas como prevenir ciberfraudes y hacer 100 por ciento seguros los cobros y pagos (Gutiérrez Julio, 2019). 
La alternativa e-commerce hace referencia al método de compra - venta de productos y servicios mediante medios electrónicos y a través de dispositivos que se conectan a la red. Se puede realizar la compra a través de un ordenador, tableta o móvil. Aunque es difícil cuantificar qué proporción del comercio total abarca el denominado e-commerce en relación con las ventas totales (se calcula que 4 por ciento), al menos 95 por ciento de usuarios con dispositivos inteligentes ocupa esa vía para consumir y ocho de cada 10 realizan compras de ese tipo. Hasta el segundo trimestre de 2019 existían 59 millones 600 mil usuarios que utilizaban esa alternativa digital para el consumo (Competitive Intelligence Unit, 2019). La misma fuente indica que al menos en los pasados cinco años el e-commerce ha crecido muy fuerte, ya que anteriormente lo más común era realizar la adquisición de un servicio para viajar, pero ahora la demanda de bienes tradicionales, desde una botella de agua hasta la despensa, se realiza por ese medio.

Esto hace necesario contar con una página de ventas y atención al cliente propia, creando una relación marca-cliente más fuerte. Aunque la UEASM ya tiene su página será necesario crear una tienda en línea que facilite esta estrategia de ventas. El diseño de la tienda en línea debe presentar diferentes pestañas, las cuales llevan de la mano al cliente, consultando los productos de su interés, sus precios, características, e información a detalle sobre los productos ofrecidos. Muela (Molina Clara, 2008) Un punto importante que se ofrece como información es la contribución y el impacto que el consumidos realiza al consumir los productos de la UEASM, en el manejo y conservación sustentable de las selvas de México, y su contribución a la economía campesina.

\section{CONCLUSIONES}

Dentro de la cadena productiva, que los ejidos de la UEASM han desarrollado, es necesario integrar la última fase del proceso, que incluye los aspectos de mercadeo, comercialización y márquetin, con el fin de cerrar la cadena y ver reflejados los ingresos por las ventas de sus productos, es por ello que la participación de la UACh es relevante aportando propuestas y capacitación a los miembros de la UEASM.

Los temas que se deben abordar en la siguiente etapa de la investigación son los siguientes:

A) Crear una colección digital de productos que ofrece la UEASM., B) Identificar y seleccionar el o lo(s) segmentos de mercado para los productos la UEASM. C) Identificar y seleccionar las mejores opciones de comercialización para los productos ofertados. D) Crear el manual de identidad de la organización (marca, logo, eslogan). E) Diseñar una estrategia digital de Social Media. F) Rediseño de la página web. G) Diseño de una tienda on line. H) Capacitar a los socios de la UEASM en el Manejo Social Media. I) Capacitar a los responsables de la comercialización en el manejo y actualización de la estrategia de mercadeo, comercialización y mercadotecnia. 


\section{REFERENCIAS}

Acta constitutiva de la Unión de Ejidos “Alianza Selva Maya de Quintana Roo (UE de RL)”. 2014.Notaria pública No. 5. Chetumal, Quintana Roo. Li. Reynaldo Vanegas Marín.

CIU. (2019). Análisis de mercado oportunidad de negocio e impacto de la regulación en telecomunicaciones. Disponible en https://www.theciu.com/

Gutiérrez Julio. (2019). Crece el comercio electrónico 15 veces más que la economía. Periódico La Jornada domingo 18 de agosto de 2019, p. 17 . Disponible en https://www.jornada.com.mx/2019/08/18/economia/017n1eco?partner=rss

http://www.edu.xunta.gal/centros/iessanchezcanton/aulavirtual2/file.php/322/Recursos/LA_PUBLICIDAD.pdf

IAB México y Millward Brown. (2018). Estudio de consumo de medios y dispositivos entre internautas mexicanos. Disponible en https://www.iabmexico.com/estudios/consumo-medios-2018/

Kotler, Philip y Gary Armstrong. (2008). Fundamentos de mercadotecnia. Pearson Educación. México D.F. 522 p.

Kotler, Philip y Gary Armstrong. (2012). Marketing. Pearson Educación. México D.F. 613 p.

Muela Molina Clara. (2008) Quiero una empresa. Zer Vol. 13 - Núm. 24 ISSN: 1137-1102 pp. 183-201. Disponible en: http://www.quieroempresa.com/wp-content/uploads/2017/09/publicidad-internet.pdf 